\title{
Effect of Proparacaine Hydrochloride Eye Drops on the Ocular Surface
}

\author{
Xiaofeng Li $i^{1, \dagger}$, Xuemei Wang ${ }^{1,2, \dagger}$, Yanzi Wang ${ }^{1,2}$, Huping Wu ${ }^{1, ~ *}$, Cheng $\mathrm{Li}^{1,2, \text { * }}$ \\ ${ }^{1}$ Eye Institute \& Affiliated Xiamen Eye Center, School of Medicine, Xiamen University, Xiamen, China \\ ${ }^{2}$ Fujian Provincial Key Laboratory of Ophthalmology and Visual Science, Xiamen, China
}

\section{Email address:}

lipipi@189.cn (Xiaofeng Li), 1904141200@qq.com (Xuemei Wang), yencn@163.com (Yanzi Wang), wuhuping123@163.com (Huping Wu), cheng-li@xmu.edu.cn (Cheng Li)

${ }^{*}$ Corresponding author

$\dagger$ Xiaofeng Li and Xuemei Wang are co-first authors.

\section{To cite this article:}

Xiaofeng Li, Xuemei Wang, Yanzi Wang, Huping Wu, Cheng Li. Effect of Proparacaine Hydrochloride Eye Drops on the Ocular Surface. International Journal of Ophthalmology \& Visual Science. Vol. 6, No. 1, 2021, pp. 46-53. doi: 10.11648/j.ijovs.20210601.17

Received: February 19, 2021; Accepted: March 4, 2021; Published: March 10, 2021

\begin{abstract}
Background: Proparacaine hydrochloride (PH), as a a local anesthetic, is used regularly in ophthalmic surgery and optometry. However, few pieces of research on the ocular surface toxicity of PH eye drops have so far been reported. Purpuse: To evaluate the effect of PH on the ocular surface of mice. Methods: Male C57/BL6 mice were divided into four groups: normal, vehicle-treated, $0.05 \%$ PH-treated and $0.5 \% \mathrm{PH}$ treated 7 days. The clinical indications were tear production, corneal sensitivity, and fluorescein staining. The structure and morphology of the cornea were examined by confocal microscopy; the thickness of the corneal center was examined by anterior segment optical coherence tomography (AS-OCT); and the corneal epithelial microvilli morphology was examined using scanning electron microscopy. Hematoxylin-eosin staining was used to characterize the central corneal morphology. Immunofluorescence staining for cytokeratin 10 (K10) was employed to evaluate squamous metaplasia in the corneal epithelium. Results: The results show that topical PH treatment diminished tear production and corneal sensitivity, and increased corneal fluorescein staining scores. Moreover, PH altered the corneal epithelial microvilli morphology, disrupted the epithelial barrier of the cornea, and promoted apoptosis of ocular surface cells. Furthermore, the expression of K10 in the corneal epithelium was found to be increased. Conclusion: Treatment with $0.5 \%$ PH caused instability of the tear film, and changes in corneal sensitivity and ocular surface homeostasis.
\end{abstract}

Keywords: Proparacaine Hydrochloride, Cornea, Ocular Surface

\section{Introduction}

The ocular surface is composed of the cornea and conjunctiva, which protect the eye against injury and ensure clear vision. The corneal epithelium is regarded as the most superficial layer of the cornea and serves as the first line of defense against external attacks. Damage to this highly specialized structure can result in loss of vision; hence, understanding of its protection mechanisms is essential [1]. The corneal epithelium also helps to regulate the immune response. If potential antigenic molecules enter deeper into the corneal structure, epithelial cells quickly stimulate immune reactions on the ocular surface; for instance, the production of the cytokine interleukin-1 (IL-1) [2]. Excessive loss of human corneal epithelial cells as a result of trauma, microbial infections, and harmful substances can cause barrier function destruction, irreversible keratopathy, visual impairment, and even blindness [3].

Proparacaine hydrochloride ( $\mathrm{PH})$ is a local anesthetic used regularly in ophthalmic surgery and optometry, owing to its excellent efficacy and rare adverse effects [4]. PH appears to bind or antagonize the function of voltage gated sodium channels, inducing a reversible nerve conduction block. This treatment inhibits depolarization of the cell membrane, thereby eliminating the sensation of the pain. However, repeated use of $\mathrm{PH}$ in the long-term has been indicated as harmful to the cornea, increasing corneal thickness and causing corneal turbidity in rabbit models [5], and damaging endothelial cells and promoting thickening of the cornea in cat 
models [6]. The most common findings associated with anesthesia abuse are corneal epithelial loss, inhibition of healing of corneal epithelial defects, stromal infiltration due to edema, and keratitis with ring infiltrates [7]. Commercial PH eye drops exist in single- and multi-dose forms; the multi-dose package contains benzalkonium chloride (BAC) as a preservative, while the single-dose package contains no preservatives. Most of the literature concerning damage to the cornea refers to that following multiple doses of $\mathrm{PH}$, while BAC toxicity toward the ocular surface has been found in both in vitro and in vivo models and in clinical studies $[8,9]$. Thus, the toxicity of PH toward the ocular surface and its underlying mechanism require further exploration.

The amount and composition of tear film are critical for the health, maintenance, and protection of the cells of the cornea and conjunctiva. Small changes in the concentration of tear electrolytes are correlated with dry eye, a multifactorial syndrome that can be caused by alteration in the quality or quantity of the tear film. Tear film stability protects the ocular surface epithelium from desiccation and is ensured via cooperation between the ocular surface components including constituents of the tear film and ocular surface epithelium. Thus, when those components are insufficient or impaired, the tear film breakup that initiates dry eye occurs. The secretory mechanisms of water, electrolytes, and proteins from the lacrimal gland differ, all three are under tight neural control. This allows for a rapid response to environmental conditions in order to meet the needs of the ocular surface cells. The neural response consists of activation of the afferent sensory nerves in the cornea and conjunctiva to stimulate efferent parasympathetic and sympathetic nerves that innervate the lacrimal gland [10]. Local anesthetics act on any part of the nervous system and on every type of nerve and fiber. Different sensations are lost based on the size of the axon serving them. Due to its moderate properties, $\mathrm{PH}$ is one of the most versatile agents used in anesthesia, and is often employed by optometrists and ophthalmologists in different clinical procedures. The mechanism by which $\mathrm{PH}$ affects ocular surface homeostasis by influencing tear secretion requires further research.

In the present study, we developed an in vivo animal model and applied a single daily dose of $\mathrm{PH}$ eyedrops without preservatives toward the mouse cornea to investigate the toxicity of PH on the ocular surface. We found that $\mathrm{PH}$ not only damaged the structure of the mouse corneal epithelium, but also decreased corneal sensitivity and disrupted tear film stability. These results may have crucial implications in the clinical treatment of dry eye.

\section{Materials and Methods}

\subsection{Animals}

The present study employed 32 SPF male C57/BL6 mice (6-8-weeks-old) (Shanghai SLAC Experimental Animal Center, China) that were housed in a clean environment during the experiment, at a temperature of $24 \pm 1^{\circ} \mathrm{C}$, a relative humidity of $59 \pm 9 \%$, and a 12 -hour light/dark cycle ( 8 am to 8 pm). All mice were sacrificed by $\mathrm{CO}_{2}$ asphyxiation followed by cervical dislocation, and the confirmation of death is assessed through direct palpation of either the pulse in the carotid or femoral artery or direct cardiac palpation. The research protocol was approved by the Experimental Animal Ethics Committee, Xiamen University (allowance number: XMUMC: 2019-12-10) and met the standards stated in the ARVO Declaration on the Use of Animals in Ophthalmology and Visual Studies.

\subsection{Local PH Treatment}

Single-dose $0.5 \%$ non-preserved $\mathrm{PH}$ eye drops (RuiNian Best, Nanjing, China) were used in the present study and diluted with non-preserved polyvinyl alcohol eye drops (Ruizhu, Hubei, China) to yield a PH solution with a final concentration of $0.05 \%$. Mice were randomly divided into four groups, each containing six mice. The first group was left untreated as the normal group. The remaining three groups received $8 \mu \mathrm{L} 0.05 \% \mathrm{PH}, 0.5 \% \mathrm{PH}$, and non-preserved polyvinyl alcohol eye drops (vehicle group), respectively, in both eyes, five times a day for seven days.

\subsection{Measurement of Tear Production and Corneal Sensitivity}

Tear production was measured forty minutes after the instillation of PH using phenol red cotton thread (Tianjin, China) (6 eyes per group). The thread was placed in the lower conjunctiva, one third of the distance from the lower eyelid, for 15 seconds. The length of the wet red line was measured (in millimeters) using the scale on the cotton thread. The test was repeated three times for each eye, and the final length was considered the average length of the red portion.

To measure the sensitivity of the central cornea in mice, a Cochet-Bonnet esthesiometer (Luneau, France) was employed 30 minutes after application of the eye drops. While holding the mouse, the nylon wire was allowed to contact the center of the cornea, and a positive response was recorded if three reflections (induced blinking) occurred in response to five consecutive touches. Measurements were taken from a fully extended $60-\mathrm{mm}$ wire that was applied with minimum pressure and gradually shortened in increments of $5 \mathrm{~mm}$, until a response was observed. The corneal sensitivity threshold was regarded as the longest filament length that caused a positive result.

\subsection{Slit-lamp Evaluation and Fluorescein Test}

A slit-lamp microscope (BQ900H Haag-Streit, Bern, Switzerland) was used to examine the eyes of the mice. Subsequently, the cornea was observed following the administration of $2 \mu \mathrm{L} 0.1 \%$ sodium fluorescein, and after blinking for a while, to ensure the accuracy of the experiment, the mice in all groups were rinsed with saline the same number of times after blinking. The appearance of the cornea was observed and graded using the cobalt blue filter. The cornea was divided into four quadrants [11], which were 
graded as: no presence - 0 ; area stained with less than 30 spots - 1; areas stained with more than 30 spots but not diffusely - 2; strongly diffuse staining but no positive plaques - 3; and positive fluorescein spots - 4. The final grade was obtained as the sum of the scores for each quadrant (16 points in total).

\subsection{In vivo Confocal Microscopy (IVCM)}

The mice were administered sodium pentobarbital (50 $\mathrm{mg} / \mathrm{kg}$ i.p., Abbott, Northern Chicago, IL) prior to examination of the corneal central structure by confocal laser scanning microscopy using a Heidelberg Retina Scanner III/Rostock Corneal Module (Heidelberg Engineer GmbH, Heidelberg, Germany), which employed a $60 \times$ immersion objective (Olympus, Hamburg, Germany) and a 670-nm diode laser. The image consisted of $384 \times 384$ pixels, providing an area of $400 \mu \mathrm{m}^{2}$ for scanning, with $1 \mathrm{~mm}$ horizontal and vertical resolutions; the maximum magnification was $800 \times$. Prior to the observation, a drop of carbomer gel (Alcon Laboratories, Fort Worth, TX) was used as a coupling medium between the flattened lens and the cornea. By adjusting the controller, the cap center was extended to the center of the cornea, with a computer screen showing a digital image of the cornea. At least 10 images were taken of each of the following structures: the superficial epithelial layer, basal epithelial layer, stromal layer, and endothelial layer. All measurements were performed by a researcher who was blinded to the specific experimental conditions.

\subsection{Anterior Segment Optical Coherence Tomography (AS-OCT)}

AS-OCT has been increasingly used for non-contact imaging of the cornea, anterior chamber angle, aqueous outflow pathway, sclera, and structures of the ocular surface. The AS-OCT (ZEISS, Germany) long-lens single-line scanning mode was selected, the Axial resolution was $18 \mathrm{~mm}$ and lateral resolution was $50 \mathrm{~mm}$, and the position of the mouse's head was adjusted. The central cornea was aligned using the in situ fixed spotlight, and the lens was positioned to ensure that the corneal image was in the center of the scanning window prior to imaging. The image was scanned continuously three times or more times. OCT analyzed the central corneal thickness data.

\subsection{Hematoxylin-eosin Staining}

After the mice were sacrificed, the eyes were removed from each group $(\mathrm{n}=4)$ and directly placed in optimal cutting temperature (OCT) components (Catalog No. 4583; SAKURA Tissue-Tek, Torrance, CA, USA), which were used for embedding. Sections $(7-\mu \mathrm{m})$ were cut in the sagittal plane at the center of the eye using a cryostat (CM1850 UV; Leica Microsystems, Wetzlar, Germany) and placed at $-80^{\circ} \mathrm{C}$. Sections were stained with hematoxylin-eosin as previously described and examined under a light microscope.

\subsection{Examination of Corneal Epithelial Microvilli Morphology}

The cornea was carefully dissected along the limbus and immediately fixed in cold $2.5 \%$ glutaraldehyde for $8 \mathrm{~h}$. Samples were then prepared for SEM [12] (S-4800; Hitachi, Tokyo, Japan) which was employed capture images for comparison.

\subsection{Immunofluorescence Staining}

Frozen sections were fixed in cold acetone at $-20^{\circ} \mathrm{C}$ for 10 $\mathrm{min}$, and then permeabilized in PBS containing $0.2 \%$ Triton $\mathrm{X}-100$ for $20 \mathrm{~min}$. Subsequently, the sections were incubated overnight at $4{ }^{\circ} \mathrm{C}$ with a primary antibody against K10 (1:200; Abcam, Inc.). After washing three times with PBS, the sections were incubated with an anti-rabbit IgG secondary antibody (1:300; Life Technologies, USA) for $1.5 \mathrm{~h}$ at room temperature in the dark. Sections were then washed three times with PBS and counterstained with 4',6-diamidino-2-phenylindole (DAPI; Cat. No. H-1200; Vector Laboratories, Inc., Burlingame, California, USA). Representative digital images of the regions were taken using a vertical microscope (DM2500; Leica Microsystems), and the fluorescence intensity was analyzed using the (Nikon software).

\subsection{Statistical Analysis}

GraphPad Prism 5 (GraphPad Software Inc., USA) was used for statistical analysis. Images were processed using Image J $1.48 \mathrm{v}$ (USA). The groups were compared using one-way ANOVA followed by Tukey's post-hoc test. Data are expressed as the mean \pm standard error (SEM). $P$ value $<0.05$ was considered statistically significant.

\section{Results}

\subsection{Corneal Fluorescence Staining}

The corneal epithelial defects in mice induced by $\mathrm{PH}$ were evaluated by corneal fluorescence staining. It was observed that sodium fluorescein staining of the cornea was not obvious after three days but gradually became apparent as the duration of $\mathrm{PH}$ treatment increased (Figure 1A). In comparison with the normal group, the fluorescein staining score was significantly higher on day 7 of PH treatment (Figure 1B).

\subsection{Tear Film and Corneal Sensitivity Changes Following PH Treatment}

Normal tear secretion maintains tear film stability and protects the corneal epithelium. There were significant differences in the tear production between the vehicle and $0.5 \%$ PH-treated eyes on day 7 (Figure 2A). To determine whether $\mathrm{PH}$ application had an effect on corneal sensitivity, the mean corneal sensitivity threshold in the vehicle eyes $(5.75 \mathrm{~mm})$ was compared with that obtained three days after $0.05 \%$ or $0.5 \% \mathrm{PH}$ application $(5.32 \mathrm{~mm}$ and $5.31 \mathrm{~mm})$. These values were not statistically different; however, significant decreases were observed following after seven days treatment of $0.5 \% \mathrm{PH}$ (Figure 2B). 
A

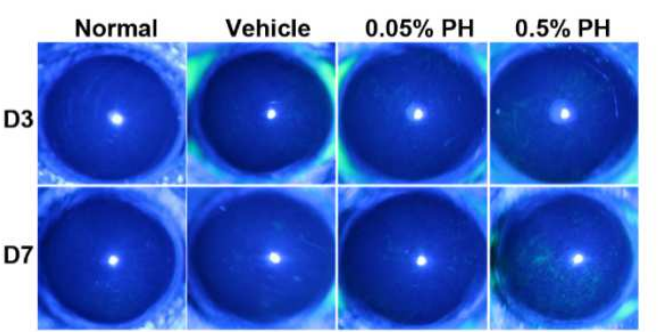

B

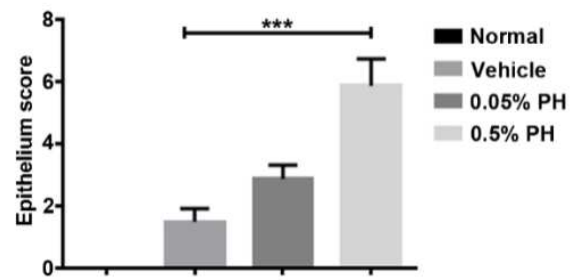

Figure 1. Clinical evaluation of the acute effects of PH on the ocular surface. (A) Representative images of fluorescein staining. (B) Fluorescein staining score on day 7. PH, proparacaine hydrochloride. Data are expressed as the mean \pm standard deviation $(n=8)$. ${ }^{* * *} P<0.001$ vs the vehicle group.
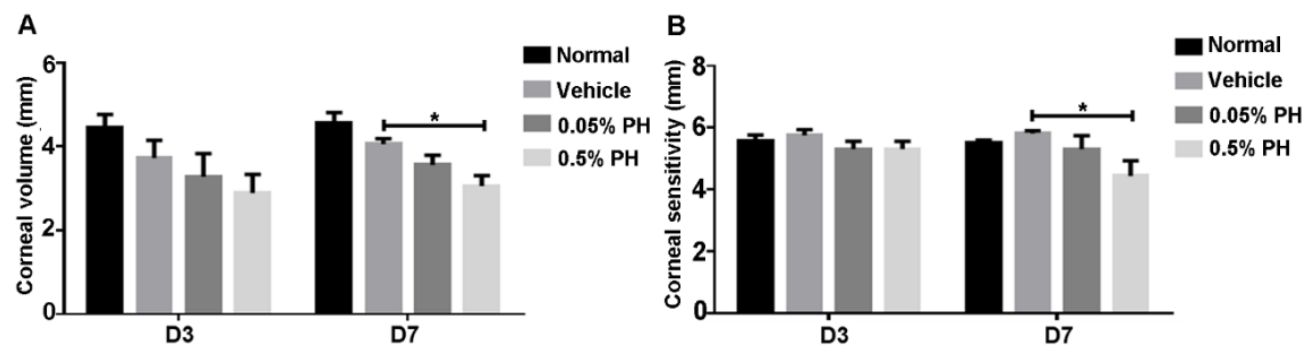

Figure 2. Tear film and corneal sensitivity change after PH treatment. (A) Tear production measured by the phenol red thread test on day 7. Data are expressed as the mean \pm standard deviation ( $n=6) .{ }^{*} P<0.05$ vs the vehicle group. (B) Corneal sensitivity score on day 7. Data are expressed as the mean \pm standard deviation ( $n=8) . * P<0.05$ vs the vehicle group.

\subsection{In Vivo Confocal Microscopy}

In normal and vehicle-treated mice, the superficial epithelial cells of the cornea had a polygonal mosaic appearance accompanied by brightly reflective nuclei. However, the superficial epithelial cells of the cornea in the eyes treated with PH exhibited various abnormalities, such as partial desquamation, abnormal shape, anisocytosis and loss of cell boundaries, irregular reflectivity patterns, and swelling. In the eyes treated with $0.5 \% \mathrm{PH}$, the superficial epithelial cells were clearly visible and significantly larger than those in the normal and vehicle-treated groups. These larger cells exhibited a bright reflective round mosaic appearance with dark nuclei (Figure 3). A regular mosaic of dark cell bodies with light, narrow inter-cellular borders was observed in the basal epithelial cells in the normal and vehicle-treated groups. However, the basal epithelial cells treated with PH had an increased cell gap, protruding nuclei, and were activated (Figure 3).

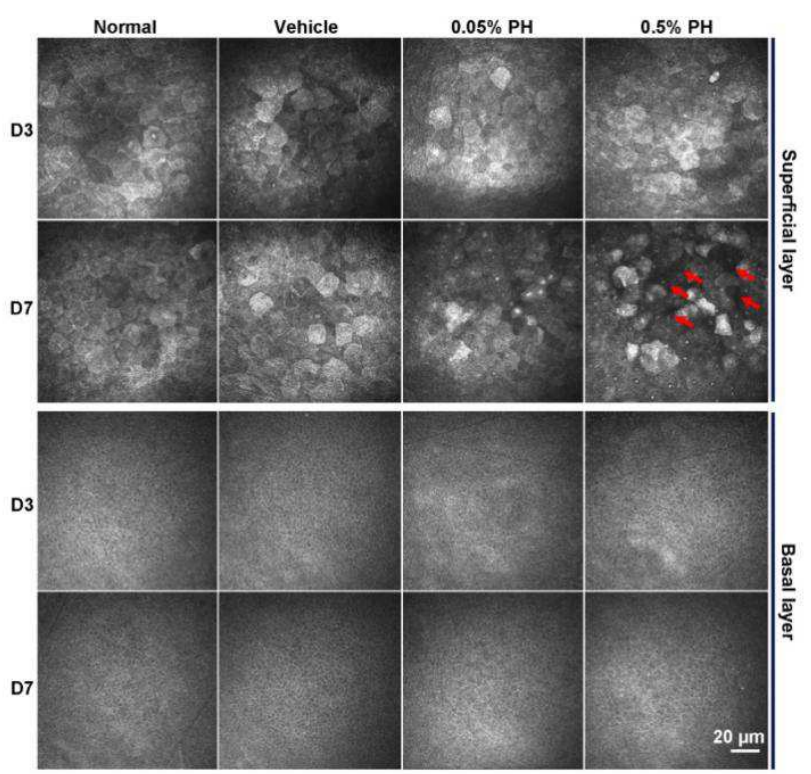

Figure 3. Toxic effects of PH on the morphology of mouse superficial corneal epithelial cells. Representative in vivo confocal images of the corneal epithelium in different groups. Eyes treated with $0.5 \%$ PH show a loss of superficial epithelial cells (dark areas, arrowheads), with blurry boundaries and abnormally reflected nuclei as compared with normal eyes. Toxic effects of PH on the morphology of the mouse corneal epithelial basal layer. Representative in vivo confocal images of the corneal epithelium in different groups. The corneal epithelial basal cells treated with PH show increased gaps and abnormally reflected nuclei as compared with those of normal and vehicle eyes. 


\section{4. $A S-O C T$}

The AS-OCT can scan the entire thickness of the mouse cornea, including the epithelial layer, the anterior elastic layer, the stromal layer, the posterior elastic layer, and the endothelial layer (Figure 4A). No significant differences were found in the central thickness of the cornea after the administration of $\mathrm{PH}$, when compared with the vehicle and normal groups (Figure 4B).
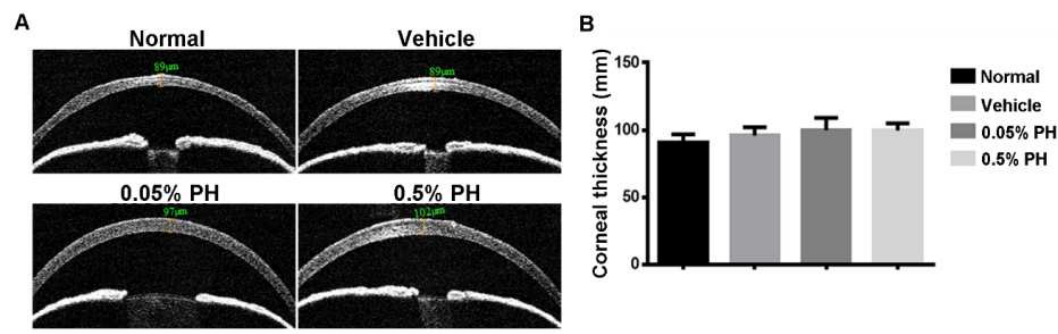

Figure 4. Toxic effects of PH on the structure of the mouse cornea. (A) Representative AS-OCT images of the cornea in different groups. (B) Corneal thickness on day 7. Data are expressed as the mean \pm standard deviation $(n=4)$. Corneal thickness was not significantly change on day 7 as compared with that in the normal group.

\subsection{Corneal Epithelial Changes Following PH Treatment}

We further examined the morphological changes in the corneal epithelium using experimental animals. Hematoxylin-eosin staining demonstrates that the corneal epithelium in the normal and vehicle-treated groups was intact and smooth. The nuclei of the superficial corneal epithelial layers in mice treated with PH were more condensed and the surface was rougher. It was observed that mice with obvious corneal fluorescence staining in the treatment group had a rough corneal epithelial surface (Figure 5A). Scanning electron microscopy revealed that the eyes treated with PH exhibited less dense and shorter microvilli (Figure 5B).

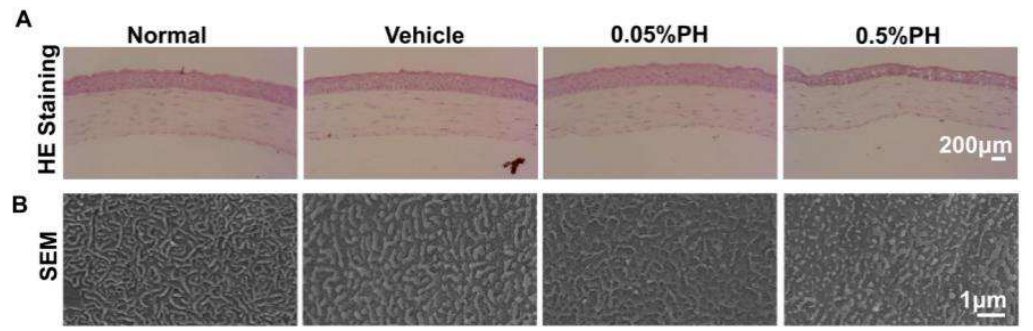

Figure 5. Histological changes in the corneal epithelium following treatment with PH. (A) Superficial layers of corneal epithelial cells treated with PH became more compact, and the corneal epithelial surface became rough. (B) Scanning electron microscopy images of the corneal epithelium in different groups on day 7. The superficial conrneal cells of vehicle eyes were normal in appearance with typical microvilli; however, the superficial cells were damaged in PH-treated eyes and exhibited degenerated microvilli. Following treatment with $0.5 \%$ PH eye drops for 7 days, corneal epithelial microvilli were shorter and disordered.

\subsection{PH-induced Phenotypic Changes}

Keratin 10 is an epidermal keratinocyte-specific intermediate filament that is not expressed in normal ocular surface epithelium. K10-positive cells in the central cornea of mice treated with PH displayed an obvious increase in K10 expression as compared with those in the normal and vehicle-treated groups (Figure 6A). In comparison with the vehicle group, the difference in K10 expression in the $0.5 \%$ PH-treated group was statistically significant $(P<0.05$, Figure $6 \mathrm{~B})$.
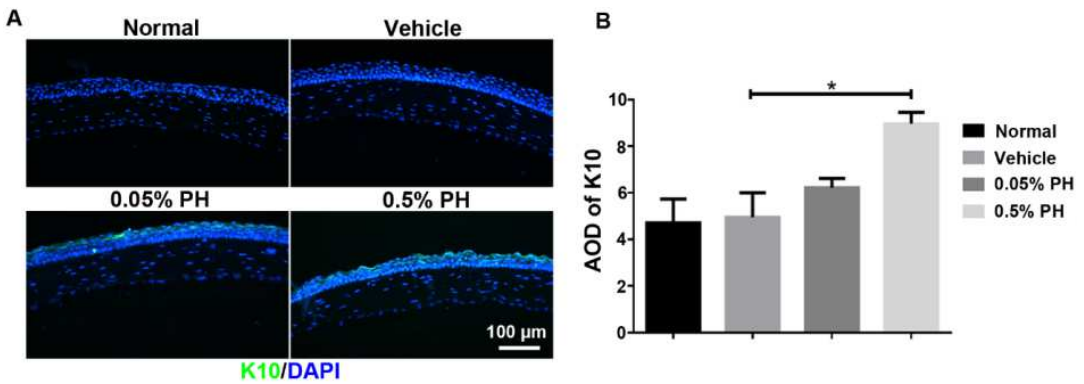

Figure 6. Squamous metaplasia in the cornea. (A) Representative images of K10 in the corneal epithelium on day 7. In comparison with the normal and vehicle groups, there was a marked upregulation of K10-positive cells in the central cornea of the PH-treated groups on day 7. (B) Average optical density (AOD) in each group. There was a significant difference between the vehicle and $0.5 \%$ PH-treated groups. Values are expressed as the mean \pm standard deviation $(n=3) . * P<$ 0.05 vs. vehicle group. 


\section{Discussion}

The cornea is located at the front of the eye, providing us with a clear view; the integrity and function of the outermost layer is critical to vision. Superficial keratitis, persistent epithelial defects, interstitial/ring infiltration, corneal edema, endothelial damage, and ocular inflammation can be caused by local anesthetic abuse, even after dilution [13]. Topical anesthetic drugs are most commonly used in daily ophthalmic practice, and overuse of these drugs by patients can result in a burning, stinging, or piercing sensation [14]. Although topical anesthetic drugs cause the cornea to lose sensation as a mechanism of pain reduction, they exhibit toxic effects, especially to the corneal epithelium, following frequent and long-term use. Pain caused by anesthetic damage may lead to an increase in the frequency with which patients use these drugs, thereby worsening the situation [15].

Several mechanisms have been proposed to explain the pathogenesis of keratopathy and the adverse effects of topical anesthetic drugs [16]. Topical anesthetic drugs have been reported to reduce the viability of cultured human corneal cells [17]. Microscopic observation of corneal endothelial cells has revealed a decrease in cell number, demonstrating its toxicity toward the endothelium [18]. The loss of endothelial cells is also thought to be a result of severe inflammation of the anterior chamber and the presence of keratin deposits [19]. An immunological interpretation of the toxic mechanism is the formation of antigen-antibody complexes that cause infiltration in the cyclic matrix. Another more likely explanation involves the role of preservatives, which may cause additional toxic effects. Benzalkonium chloride, the most commonly used preservative has significant toxic effects on ocular tissues [20]. The innovation of this study lies in the application of preservative-free eye drops in animal models, and it is found for the first time that anesthetics can induce corneal epithelial squamous metaplasia.

The microvilli in normal corneal epithelial surface cells help corneal epithelium to adhere to the various components in the tear film, especially the membrane-bound mucin secreted by corneal epithelial cells, such as Muc1, Muc4, and Muc16. Due to the destruction of microvilli in epithelial cells, topical anesthetic drugs can cause tear film instability and a decreased mucus adhesion by reducing the production of water-reflective tears. The instability of the tear film, reduction in reflex tears, and reduction in blink speed result in the evaporation of tears at an increased rate, leading to dry eye and epithelial damage. Innervation is crucial in the maintenance of corneal structure and function. The neuropeptides present in the corneal nerve may be involved in these effects. Studies have shown that trigeminal and sympathetic neurons regulate the proliferation and differentiation of epithelial cells in vitro. The nerve of the cornea delivers blink reflexes to maintain a sufficient ocular hydration, and also secretes neuropeptides that are mitotic to surrounding epithelial cells. In this respect, corneal epithelial nerves and nerve endings, including the underlying layer, are particularly crucial. The nerve endings include the whole epithelial axon at the lower end of the basal nerve, as well as all of its collateral and distal expansions [21]. The nerve endings are regarded as the parts of nerve fibers that play a role in transmitting sensory stimuli and passing on the nerve signal; therefore, the corneal nerve ending density is closely related to corneal sensitivity. Depletion of the corneal nerve leads to neurotrophic keratitis, a clinical symptom accompanied by corneal anesthesia and dehydration, with irregular epithelial metabolism [22]. In addition, research has revealed that certain diseases, such as diabetes, keratitis, and dry eye, are associated with corneal nerves [23]. Erdelyi et al. studied the sub-corneal basal nerve in rabbits with dry eye using confocal laser scanning microscopy and found that the sub-basal nerve density was markedly lower than that in normal controls [24]. Corneal sensitivity is clinically related to the density and quantity of nerve endings [23]. It is reported that nerve terminal and sub-basal nerve loss may contribute to the corneal sensitivity decrease. In the present study, it was found that corneal sensitivity was significantly reduced in the $0.5 \% \mathrm{PH}$-treated group as compared with that in the normal group. However, it is known that corneal nerves and nerve endings with diameters $<0.5 \mathrm{~mm}$ may not be captured during IVCM [25]. As a result, we failed to obtain clear images of the changes in corneal nerve endings by IVCM.

Studies on the cellular and molecular mechanisms supporting the pathogenesis of corneal epithelial diseases, such as neurotrophic keratopathy, have clarified the critical role played by the trigeminal nerve in maintaining corneal health and function. The corneal nerve not only protects the ocular surface through complex sensory and blink reflex mechanisms, but also releases various trophic factors and regulates epithelial integrity, proliferation, and wound healing [26, 27]. Epithelial metabolism is also disrupted due to impairment of nutritional function in corneal nerve fibers; thus, we propose that $\mathrm{PH}$ damages the corneal epithelium by affecting epithelial nerve fibers [19]. The results of previous studies have shown that a single drop of $\mathrm{PH}$ in the eye produces a significant anesthetic effect for up to 15 minutes, but multiple infusions can prolong the anesthesia [28]. The blinking mechanism protects the ocular surface from external harmful stimuli and spreads tears evenly. Blinking is regarded as vital for maintaining the integrity of the ocular surface by preservation of moisture, tearing, secretion of lipids from the meibomian glands, and diffusion of tear lipids on the pre-corneal tear film. In the eyes, during spontaneous full blinking, the upper and lower eyelids, which are lubricated by the tear film, can move relative to the eyeball and corneal surface. This blinking action plays a crucial role in creating a smooth optical surface through reformation of the tear film, which undergoes a destabilization process at the interconnecting interval. Blinking also removes the excess debris that remains in the tear film and helps to clear it through tears. Although blinking is important for optical performance, maintenance of ocular surface health, and tear discharge, if the tear film is insufficient, the mechanical force of the blink may injure the wiper and/or ocular surface (the wiper is behind the 
central eye; the part of the posterior eyelid that is juxtaposed with the ocular surface) [29]. Topical anesthesia reduces the rate of spontaneous blinking [30]; therefore, we suspect that $\mathrm{PH}$ may reduce the number of blinks, resulting in damage to the corneal epithelial cells. Unfortunately, we failed to measure changes in blinking following topical administration of $\mathrm{PH}$.

Squamous metaplasia is considered a hallmark of various serious ocular surface diseases, including chronic dry eye, and often occurs in the long-term absence of tear secretion and chronic inflammatory infiltration of the ocular surface [31]. The present study found that corneal epithelium treated with $\mathrm{PH}$ specifically expressed K10, demonstrating that the unkeratinized epithelium was disrupted by the squamous epithelium. The corneal epithelium is the thin outer layer of the cornea that protects the eye against the external environment and maintains maximum optical quality by supplying a smooth front surface. Although the cells of normal eyes continue to flow, the corneal epithelium thickness is extremely uniform and maintains a stable contour. Measuring the epithelial layer thickness of the cornea and its behavioral characteristics in response to changes in corneal structure have attracted increasing attention in the clinic. The epithelium has a significant remodeling ability that responds to changes in the underlying interstitial pathology or pre-corneal curvature. Advances in human corneal epithelial measurement techniques have led to substantial developments in the characterization of the pathological behavior of the cornea (such as keratoconus), screening for refractive surgery candidates, and understanding the normal, pathological and postoperative epithelial remodeling processes [32]. In the present study, no significant difference was found in the central corneal thickness following treatment with $0.5 \% \mathrm{PH}$.

It was observed that treatment with $0.5 \% \mathrm{PH}$ resulted in pathophysiological changes in the ocular surface of mice. The underlying mechanism of the effect for $\mathrm{PH}$ on corneal epithelial nerve function requires further study. The progressive changes described in patients with dry eye syndrome occur in the corneal epithelium within seven days. Furthermore, other pathological changes include significantly reduced aqueous tear secretion, lower corneal sensitivity, epithelial desquamation in the superficial layer, decreased apical microvilli on the surface of the corneal epithelium, corneal epithelial cell apoptosis, impaired tight junction integrity in the superficial layer, and squamous metaplasia.

We observed that PH-treated eyes exhibited certain special pathological features. But we failed to detect changes in goblet cell density in our animal model. Further studies are required to observe the differentiation of goblet cells during longer periods of treatment to investigate the effect of $0.5 \%$ $\mathrm{PH}$.

\section{Conclusion}

We clearly demonstrate that $\mathrm{PH}$ can induce injury to the ocular surface. After seven days of $\mathrm{PH}$ treatment, there was a moderate decrease in the tear volume and the the number of microvilli on corneal epithelium, the percentage of apoptotic or irregular epithelial cells increased, and even the squamous metaplasia was induced. Our findings will likely contribute to the clinical diagnosis and treatment of PH-related ocular surface diseases.

\section{Acknowledgements}

This study was supported in part by grants from the National Key R\&D Program of China (2020YFA0908100, 2018YFA0107301), the National Natural Science Foundation of China (NSFC No. 82070931, 81770891, and 81672955), the Health Research Talent Training Program of Fujian PRC (2016-ZQN-90), and the Huaxia Translational Medicine Fund for Young Scholars (No. 2017-A-001).

\section{References}

[1] Bashir H, JT Seykora, V Lee. (2017). Invisible Shield: Review of the Corneal Epithelium as a Barrier to UV Radiation, Pathogens, and Other Environmental Stimuli. J Ophthalmic Vis Res, 12 (3): 305-311.

[2] Kurpakus-Wheater M, KA Kernacki, LD Hazlett. (2001). Maintaining corneal integrity how the "window" stays clear. Prog Histochem Cytochem, 36 (3): 185-259.

[3] Loma P, A Guzman-Aranguez, MJ Perez de Lara, J Pintor. (2015). Diadenosine tetraphosphate induces tight junction disassembly thus increasing corneal epithelial permeability. $\mathrm{Br}$ J Pharmacol, 172 (4): 1045-58.

[4] Kilic A, B Gurler. (2006). Subtenon lidocaine vs topical proparacaine in adult strabismus surgery. Ann Ophthalmol (Skokie), 38 (3): 201-6.

[5] Judge AJ, K Najafi, DA Lee, KM Miller. (1997). Corneal endothelial toxicity of topical anesthesia. Ophthalmology, 104 (9): 1373-9.

[6] Wen Q, TJ Fan, SR Bai, YL Sui. (2015). Cytotoxicity of proparacaine to human corneal endothelial cells in vitro. $\mathrm{J}$ Toxicol Sci, 40 (4): 427-436.

[7] Rosenwasser GOD, S Holland, SC Pflugfelder, M Lugo, DG Heidemann, WW Culbertson, H Kattan. (1990). Topical Anesthetic Abuse. Ophthalmology, 97 (8): 967-972.

[8] Sarkar J, S Chaudhary, A Namavari, O Ozturk, JH Chang, L Yco, S Sonawane, V Khanolkar, J Hallak, S Jain. (2012). Corneal Neurotoxicity Due to Topical Benzalkonium Chloride. Invest Ophth Vis Sci, 53 (4): 1792-1802.

[9] Rasmussen CA, PL Kaufman, JA Kiland. (2014). Benzalkonium Chloride and Glaucoma. J Ocul Pharmacol Th, 30 (2-3): 163-169.

[10] Rocha EM, M Alves, JD Rios, DA Dartt. (2008). The aging lacrimal gland: Changes in structure and function. Ocul Surf, 6 (4): $162-174$.

[11] Pauly A, F Brignole-Baudouin, A Labbe, H Liang, JM Warnet, C Baudouin. (2007). New tools for the evaluation of toxic ocular surface changes in the rat. Invest Ophth Vis Sci, 48 (12): 5473-5483. 
[12] Zhang LY, DL Zou, SM Li, JQ Wang, YLW Qu, SK Ou, CK Jia, J Li, H He, TT Liu, J Yang, YX Chen, ZG Liu, W Li. (2016). An Ultra-thin Amniotic Membrane as Carrier in Corneal Epithelium Tissue-Engineering. Sci Rep-Uk, 6.

[13] Tembo AC, V Parker, I Higgins. (2013). The experience of sleep deprivation in intensive care patients: Findings from a larger hermeneutic phenomenological study. Intens Crit Care Nur, 29 (6): 310-316.

[14] Noguti J, TA Alvarenga, P Marchi, CTF Oshima, ML Andersen, DA Ribeiro. (2015). The influence of sleep restriction on expression of apoptosis regulatory proteins $\mathrm{p} 53, \mathrm{Bcl}-2$ and $\mathrm{Bax}$ following rat tongue carcinogenesis induced by 4-nitroquinoline 1-oxide. J Oral Pathol Med, 44 (3): 222-228.

[15] Park B, IS Lee, SW Hyun, K Jo, TG Lee, JS Kim, CS Kim. (2018). The Protective Effect of Polygonum cuspidatum (PCE) Aqueous Extract in a Dry Eye Model. Nutrients, 10 (10).

[16] Varga JH, RS Rubinfeld, TC Wolf, RD Stutzman, KA Peele, WS Clifford, W Madigan. (1997). Topical anesthetic abuse ring keratitis: Report of four cases. Cornea, 16 (4): 424-429.

[17] Moreira LB, N Kasetsuwan, D Sanchez, SS Shah, L LaBree, PJ McDonnell. (1999). Toxicity of topical anesthetic agents to human keratocytes in vivo. J Cataract Refr Surg, 25 (7): 975-980.

[18] Webber SK, GL Sutton, MA Lawless, CM Rogers. (1999). Ring keratitis from topical anaesthetic misuse. Aust Nz J Ophthalmol, 27 (6): 440-442.

[19] Hou YC, IJ Wang, FR Hu. (2009). Ring Keratitis Associated With Topical Abuse of a Dilute Anesthetic After Refractive Surgery. J Formos Med Assoc, 108 (12): 967-972.

[20] Eleftheriadis H, M Cheong, S Sandeman, PP Syam, P Brittain, GK Klintworth, A Lloyd, C Liu. (2002). Corneal toxicity secondary to inadvertent use of benzalkonium chloride preserved viscoelastic material in cataract surgery. Brit J Ophthalmol, 86 (3): 299-305.

[21] Marfurt CF, J Cox, S Deek, L Dvorscak. (2010). Anatomy of the human corneal innervation. Exp Eye Res, 90 (4): 478-492.

[22] Muller LJ, CF Marfurt, F Kruse, TMT Tervo. (2003). Corneal nerves: structure, contents and function. Exp Eye Res, 76 (5): 521-542.

[23] Wang C, T Fu, CY Xia, ZJ Li. (2012). Changes in Mouse Corneal Epithelial Innervation with Age. Invest Ophth Vis Sci, 53 (8): 5077-5084.

[24] Erdelyi B, R Kraak, A Zhivov, R Guthoff, J Nemeth. (2007). In vivo confocal laser scanning microscopy of the cornea in dry eye. Graef Arch Clin Exp, 245 (1): 39-44.

[25] Dvorscak L, CF Marfurt. (2008). Age-related changes in rat corneal epithelial nerve density. Invest Ophth Vis Sci, 49 (3): 910-916.

[26] Patel DV, M Tavakoli, JP Craig, N Efron, CNJ McGhee. (2009). Corneal Sensitivity and Slit Scanning In Vivo Confocal Microscopy of the Subbasal Nerve Plexus of the Normal Central and Peripheral Human Cornea. Cornea, 28 (7): 735-740.

[27] Cruzat A, Y Qazi, P Hamrah. (2017). In Vivo Confocal Microscopy of Corneal Nerves in Health and Disease. Ocul Surf, 15 (1): 15-47.

[28] Binder DR, IP Herring. (2006). Duration of corneal anesthesia following topical administration of $0.5 \%$ proparacaine hydrochloride solution in clinically normal cats. Am J Vet Res, 67 (10): 1780-1782.

[29] Pult H, SGP Tosatti, ND Spencer, JM Asfour, M Ebenhoch, PJ Murphy. (2015). Spontaneous Blinking from a Tribological Viewpoint. Ocul Surf, 13 (3): 236-249.

[30] Naase T, MJ Doughty, NF Button. (2005). An assessment of the pattern of spontaneous eyeblink activity under the influence of topical ocular anaesthesia. Graef Arch Clin Exp, 243 (4): 306-312.

[31] Murube J, L Rivas. (2003). Impression cytology on conjunctiva and cornea in dry eye patients establishes a correlation between squamous metaplasia and dry eye clinical severity. Eur J Ophthalmol, 13 (2): 115-127.

[32] Hwang ES, JM Schallhorn, JB Randleman. (2020). Utility of regional epithelial thickness measurements in corneal evaluations. Surv Ophthalmol, 65 (2): 187-204. 